\title{
THE WINNERS OF THE BEST/GOOD PAPER AWARDS 2014
}

It is our great pleasure to announce once again the winners of the Best/Good Paper Awards 2014. The winners were selected by the Editorial Team for LASER THERAPY who screened the papers in the Volume 23, 2014 issues. We would like to congratulate the selected winners by presenting them with their prize-money and certificates.

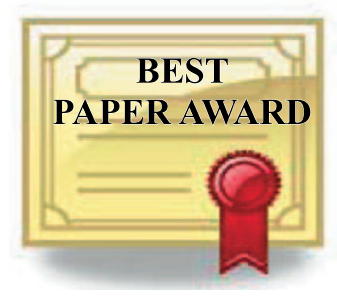

Reduction of Il-20 Expression in Rheumatoid Arthritis by Linear Polarized Infrared Light Irradiation

Asayo Imaoka, Lin Zhang, Noboru Kuboyama, Yoshimitsu Abiko

GOOD PAPER AWARDS

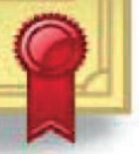

Ming Chien Kao AWARDs

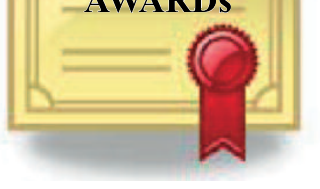

Effect of Stress-free Therapy on Cerebral Blood Flow: Comparisons among patients with metabolic cardiovascular disease, healthy subjects and placebo-treated subjects

$$
\text { Kenji Ryotokuji, Keisou Ishimaru, Kazuhiko Kihara, Yoshibisa Namiki, }
$$
Takuma Nakashima, Satoru Otani

Ex vivo efficacy evaluation of laser vaporization for treatment of benign prostatic hyperplasia using a 300-W high-power laser diode with a wavelength of $980 \mathrm{~nm}$

Junya Takada, Noribiro Honda, Hisanao Hazama, Kunio Awazu

V Treatment of Vaginal Relaxation Syndrome with an Erbium: YAG Laser Using $90^{\circ}$ and $360^{\circ}$ Scanning Scopes: A Pilot Study \& Short-term Results

Min Seok Lee

Bactericidal effect of visible light in the presence of erythrosine on Porphyromonas gingivalis and Fusobacterium nucleatum compared with diode laser, an in vitro study

Ghanbari Habiboallah, Zakeri Mahdi, Naderi Nasab Mabbobeh, Zareian Jahromi Mina, Faghibi Sina, Zakeri Majid

Low Level Laser Therapy for chronic knee joint pain patients

Takashi Nakamura, Satoru Ebihara, Ikuko Obkuni, Hideaki Izukura, Takashi Harada, Nobuyuki Ushigome, Toshio Obshiro, Yoshiro Musha, Hiroshi Takahashi, Kazuaki Tsuchiya, Ayako Kubota 


\section{BEST/GOOD PAPER AWARDS}

\section{MESSAGES FROM THE WINNERS}

\section{BEST PAPER AWARD}

\section{Asayo Imaoka,}

It is a great honor that our paper entitled "Reduction of IL-20 Expression in Rheumatoid Arthritis by Linear Polarized Infrared Light Irradiation" has been selected to the 2014 Best Paper Award of "Laser Therapy". We would like to express our sincere thanks to editorial board of "Laser Therapy". Despite a lack of consensus of the mechanism of laser therapy, we understand that laser therapy is effective in relieving pain and inflammation of rheumatoid arthritis. The reason underlying the biological mechanisms of effectiveness of laser therapies is not well elucidated especially molecular biological level. We succeeded in set up facilities of functional genomics including, DNA microarray supported from "Academic Frontier" Project for Private Universities: from Japanese Ministry of Education, Culture, Sports, Science and Technology. This grant made us possible to start a research project of the molecular biological mechanism of laser therapy by the transcriptomics conjunction with the bioinformatics. This awarded paper is a fruit of this research projects. We would like to take this opportunity to appreciate to Japanese Ministry of Education, Culture, Sports, Science and Technology along. We all deeply appreciate to your thoughtfulness in designating us for this award.

\section{GOOD PAPER AWARD}

\section{Kenji Ryotokuji,}

We are delighted to have this opportunity to express our appreciation for the receiving of the Good Paper Award 2014. We are also most grateful to the members of the International Editorial Board and the Editorial Team of Laser Therapy who recommended our paper to such a wonderful award.

In this paper, our research group firstly reported that the pinpoint plantar long-wavelength infrared light irradiation (PP-LILI) produces an elevation of the cerebral blood flow, peripheral deep-body temperature, a stabilization of blood pressure, and also significant reductions in the secretions of stress hormones such as adrenocorticotropic hormone (ACTH) and glucocorticoid (cortisol), as well as in salivary amylase. These results may possibly ameliorate the symptoms caused by stress such as diet therapy and exercise therapy in patients with type 2 (noninsulin-dependent) diabetes mellitus (DM). Based on this hypothesis, the present study was performed to examine the effects of PP-LILI on these stress hormones, blood glucose, HbA1c, insulin levels and cerebral blood flow in 10 patients with type $2 \mathrm{DM}$. Each patient received PP-LILI to the foot for 15 minutes once a week using the stress-free therapy apparatus. PP-LILI clearly has the potential for a widespread use as stress-free therapy. We currently plan to reveal the effectiveness of the present stress-free therapy through larger-scale clinical studies.

\section{GOOD PAPER AWARD}

\section{Junya Takada,}

First of all, we take this opportunity to express our sincere gratitude and appreciation to all the people who have supported our study and the Editorial Board Members of LASER THERAPY for allowing us win this award. We feel honored to receive the prestigious GOOD PAPER AWARD 2014 from LASER THERAPY.

In our article entitled "Ex vivo efficacy evaluation of laser vaporization for treatment of benign prostatic hyperplasia using a 300-W high-power laser diode with a wavelength of $980 \mathrm{~nm}$ ”, we have conducted ex vivo experiment using extracted prostate tissues to investigate the depth of vaporization and coagulation of novel high-power $980 \mathrm{~nm}$ laser diode. The treatment efficacy of this laser diode for benign prostatic hyperplasia (BPH) was quantitatively evaluated. We hope our findings will not only contribute toward the development of laser therapy for BPH, but also promote the approval of medical devices for clinical application. We will continue to make the efforts for the advancement of safer and more effective laser therapy.

We gratefully acknowledge the support for this research by all our contributors once again as an end of this letter. Thank you! 


\section{MING CHIEN KAO AWARD}

\section{Min Seok Lee}

I would like to thank the adjudication panel of the journal, Laser Therapy for awarding me the Ming-Chien Kao Award, and I express my deep gratitude to Professor Ming-Chien Kao for so generously putting up the award. It is such an privilege to win this prize. I never thought I would win such an honor.

As you know, Vaginal Relaxation Syndrome (VRS) is caused by childbirth and aging, and there are a variety of treatment options for this condition and associated problems, such as stress urinary incontinence (SUI). It was my aim to develop a novel therapeutic approach which was noninvasive and nonsurgical. My paper showed the short term clinical effect of Er:YAG laser treatment of VRS. However, a long term follow up is required for future studies. I hope more case studies are reported, based on good randomized and controlled studies, to develop solid evidence-based medicine for this new approach to improve the quality of life of affected women worldwide.

Last but not least, I would like to express my sincere gratitude to Dr. Glen Calderhead and Lutronic Corp for their dedicated support.

\section{MING CHIEN KAO AWARD}

\section{Ghanbari Habiboallah}

I, Mahdi Zakeri, as corresponding author on behalf of our research team and the all authors are proud to receive the outstanding Ming-Chien Kao Award which would be a great asset of our research resume. We believe that this award will encourage us to increase our efforts and scientific contributions and thus, develop the further investigation in this field and we hope to publish my next article in the outstanding Journal of Laser Therapy. We have to appreciate Dr. Toshio Ohshiro's collaboration as Editor-in-Chief and also his valuable help in this matter. At the end, we wish best luck and success in all dimensions for scientific team of Journal of Laser Therapy. Again, we are delighted and we want to share our happiness with our family.

\section{MING CHIEN KAO AWARD}

\section{Takashi Nakamura}

It was a privilege to receive the good paper award 2014 for our report, entitled "Low Level Laser Therapy for chronic knee joint pain patients".

It appeared that LLLT was effective treatment for chronic pain of locomotive organs. On behalf of authors, I would like to express our thanks to Professor Toshio Ohshiro (Editor-in- Chief) and editorial board members of Laser Therapy. 


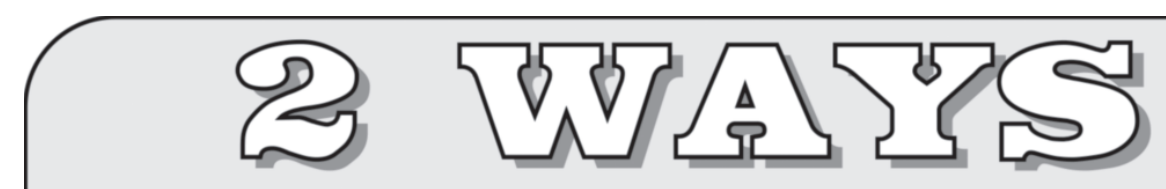

through which sharing your clinical and research experiences with Laser Therapy readers will not only earn you scientific recognition, but

\section{could earn you $¥ \$ \neq \$ \neq \$=\$ \$ \$$}

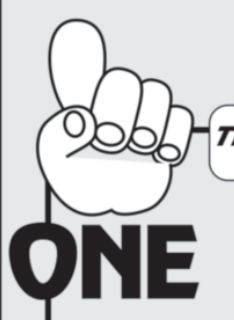

THE OHSHIRO - LASER THERAPY AWARDS

Toshio Ohshiro MD PhD, the Founding Editor and current Editor-in-Chief of Laser Therapy, in conjunction with the journal, is offering three awards; one for the best paper and two for good papers published in Volume 24 of the journal (2015), as follows: Best Paper (I) . . . JPY $¥ 500,000$ * Good Papers (2) . .JPY $¥ 2,00,000$ * each

*Awards will be paid in Japanese yen

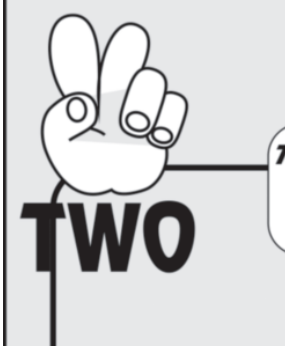

THE MING-CHIEN KAO AWARDS for YOUNG RESEARCHERS

Professor Ming-Chien Kao MD DMSc, President of the the Laser Education and Research Foundation, Tawian, ROC, is offering three awards of USD \$1000 each

to first authors who are under the age of 45 and whose clinical or research article has been accepted for publication in Volume 24 (2015) of Laser Therapy

\section{You've got to be in it to win it!!}

Get your article in to take part ... ... Start writing! 